\title{
Towards Scalable Complexity: Tools to Improve Analytical Capacity of Cancer Migration Studies
}

William J. Ashby*, Philip C. Samson**, John P. Wikswo**, Ying-Jun Su***, Tammy SobolikDelmaire***, Ann Richmond***, and Andries Zijlstra*

*Vanderbilt University Medical Center Pathology Department

**Vanderbilt Institute for Integrative Biosystems Research and Education, ***Vanderbilt University Medical Center Cancer Biology

Correlating in vitro migration studies with in vivo studies remains challenging due to the increase in complexity and parameters and loss of analytical capacity when transitioning to in vivo experiments. To address this problem we have developed a series of tools to maximize analytical capacity of microscopic analysis in vitro and in vivo. By enabling control of physiologicallyrelevant parameters in vitro and complexity in avoiding as many unknowns as possible while in order to accelerate translational research. To achieve scalable complexity of the analysis of cell migration, we have developed tools that fill gaps between in vitro and in vivo models of cancer cell migration: 1) Open-face micro-Fluidic Array (OFArray), 2) Magnetically Attachable Stencils (MAtS), 3) Murine Mammary Windows (MMW).

The OFArray enables cell loading into open-faced microfluidic devices which are subsequently sealed with glass cover slips via magnetic force. 2) MAtS pattern cell populations with micron precision while simultaneously protecting substrates enabling analysis of cell migration due to cell-cell and cell-matrix interactions, soluble factors, and cell autonomous properties. 3) MMWs: Current murine mammary-windows become obscured by the encroaching skin. MMWs trap skin in a pocket that circles the imaging area preventing encroachment into the imaging area.

These 3 tools make possible the elimination of unknowns and fill gaps between in vitro and in vivo models of cancer cell migration. By enabling scientists to incrementally add experimental complexity, the OFArray, MAtS, and MMWs represent a basis for scalable complexity with the potential to accelerate translational research. 


\section{Scalable Complexity}

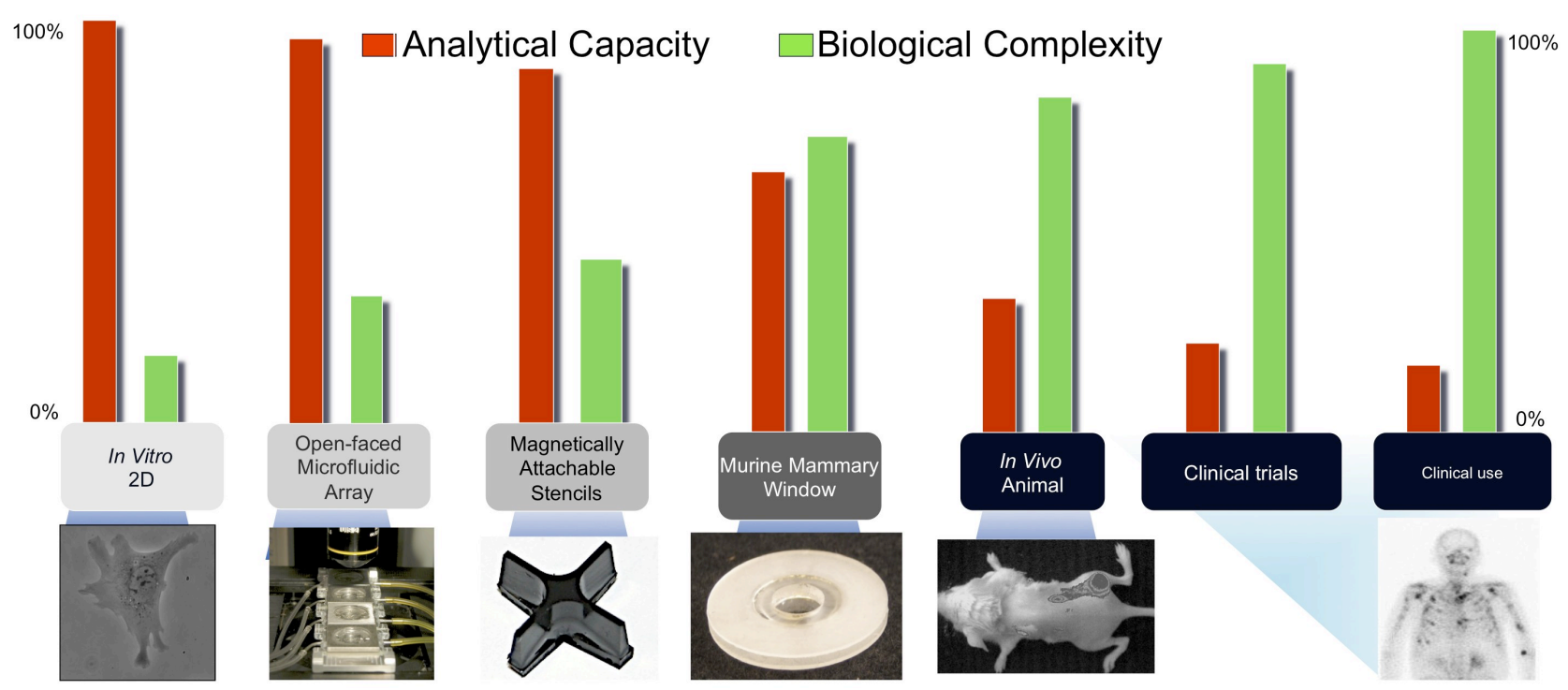

FIG. 1. Filling gaps between in vitro and in vivo models. Cell culture and mouse models are some of the most common tools used to study cancer and other illnesses. When translating research from in vitro to in vivo models, the complexity of in vivo biology often results in unexpected outcomes. To better understand and predict in vivo responses it is necessary to add complexity to in vitro systems and to improve analytical capacity of in vivo models. Using state-of-the-art microfabrication and microfluidic technology, we have created an open-faced microfluidic array, magnetically attachable stencils, and murine mammary windows that add controlled complexity to simple cell culture and improve the ability to observe in vivo models of cancer 